Pacific Journal of Mathematics

INNER FUNCTIONS: NONINVARIANT CONNECTED 


\section{INNER FUNCTIONS: NONINVARIANT CONNECTED COMPONENTS}

\section{NESTORIDIS}

$H$ denotes the family of all inner functions $B$, such that for every $\theta \in] 0,1$ [ the pseudo-hyperbolic diameters of the connected components of the set $\Sigma_{B, \theta}=\{z:|B(z)|<\theta\}$ are less than $\delta_{B, \theta}<1$.

Family $H$ is open-closed in the space of the inner functions under the uniform topology. The main result states that for every $B \in H$ the connected component of $B$ contains neither proper multiples of $B$ nor proper divisors of $B$. A characterization of the elements of $H$ is given, which in particular implies that if the zeros $\alpha_{n}, n=1,2, \cdots$ of an infinite Blaschke product $B$ satisfy condition

$$
\lim _{n} \prod_{m \neq n}\left|\frac{\alpha_{n}-\alpha_{m}}{1-\bar{\alpha}_{n} \alpha_{m}}\right|=1 \text { then } B \in H .
$$

Introduction. This work deals with the connected components of the space $F$ of the inner functions of one complex variable, under the uniform topology (see [4]). It is known ([7]) that, for $z$ the identity function $z(a)=a$, there exists an inner function $f$ belonging to the same component as $z f$. In this case the component of $f$ is invariant (under multiplication by $z$ ). There are also noninvariant connected components; the trivial examples are those of the finite Blaschke products. The existence of non trivial nonivariant components can be derived from the proof of the Theorem 1.1 in [5].

Here, we present a family of noninvariant components, larger than the family in [6]. For this purpose we consider the family $H$ of all inner functions $f$ such that, for every $\theta \in] 0,1[$, the pseudohyperbolic diameters of the connected components of the set $\Sigma_{f, \theta}=$ $\{z:|f(z)|<\theta\}$ are less than a number $\delta_{f, \theta}<1$.

A first remark is that $H$ contains only Blaschke products. Second we note that the way in which $H$ is defined permits us to apply Rouche's theorem in the case $\|f-g\|<1$ with $f \in H$. We conclude that the zeros of $f$ and $g$ are not far (one to one) in pseudo-hyperboilc distance. This yields a necessary condition for two inner functions $f, g$ to belong to the same component, when $f \in H$. Using this condition we prove that the component of each element of $H$, is not invariant. This result states precisely that for every $B \in H$, the component of $B$ contains neither proper multiples of $B$, nor proper divisors of $B$. 
The above results together with the fact that $H$ is open-closed are contained in $\S 2$. In $\S 3$, we identify the elements of $H$ as the products, in a special sense, of finite Blaschke products. In particular if the zeros $a_{n}, n=1,2, \cdots$, of an infinite Blaschke product $B$ satisfy the condition

$$
\lim _{n} \prod_{m \neq n}\left|\frac{a_{n}-a_{n}}{1-\bar{a}_{n} a_{m}}\right|=1 \text {, then } B \text { belongs to } H \text {. }
$$

We recall the information in $\S 1$ about inner functions, pseudohyperbolic distance, and the family $H$.

The family $H$ furnishes examples of noninvariant components; but the problem of identifying all noninvariant (or invariant) components seems to be open.

Finally, I wish to acknowledge my indebtedness to A. Bernard for the directions he gave me, and to D. Marshall, who answered several questions related to this work, and helped me very much with frequent discussions. To S. Negrepontis, I would like to express my gratitude for contributing an essential idea and for the encouragement he gave me.

1. Preliminaries. A: Inner Funcitons. A holomorphic function $f$, bounded on the open unit disk $D$ of the complex plane is called inner if its boundary values $f\left(e^{i \theta}\right)=\lim _{r \rightarrow 1} f\left(r e^{i \theta}\right)$ have almost every where absolute value one: $\left|f\left(e^{i \theta}\right)\right|=1$ a.e. See [3] or [8].

We will use the following notations: $b_{0}(z)=z$ and $b_{\alpha}(z)=\bar{\alpha} /|\alpha|$ $(\alpha-z) /(1-\bar{\alpha} z)$ for $\alpha \in D, \alpha \neq 0$. Therefore, a Blaschke product will have the form $c \prod_{i \in I} b_{\alpha_{i}}$, with $c$ a constant of modulus one $(|c|=1)$ and $\alpha_{i} \in D$ such that $\Sigma_{i \in I} 1-\left|\alpha_{i}\right|<\infty$.

We reserve the letter $S$ for singular functions, that is, inner functions not vanishing at any point of $D$. It is well known that every inner function $f$ can be represented as the product of a Blaschke product $B$ and a singular function $S: f=B S$.

We denote by $F$ the space of the inner functions with the uniform topology. The topology in $F$ is compatible with the following metric: $d(f, g)=\|f-g\|=\sup |f(z)-g(z)|$.

It is well known that the connected component of $z^{n}(n=0,1, \cdots)$ is the set of the finite Blaschke products with exactly $n$ zeros counting multiplicity.

B. Pseudo-hyperbolic distance. We denote by $\rho$ the pseudohyperbolic distance on the open unit disk $D$ : 


$$
\rho(x, y)=\left|\frac{x-y}{1-\bar{x} y}\right|=\left|b_{x}(y)\right| .
$$

The distance $\rho$ satisfies the following two relations:

$$
\lim _{|y| \rightarrow 1} \rho(x, y)=1 \quad \text { and } \quad \rho(x, z) \leqq \frac{\rho(x, y)+\rho(y, z)}{1+\rho(x, y) \rho(y, z)} .
$$

For $t \in] 0,1[$ and $n=1,2, \cdots$, we denote:

$$
\begin{aligned}
C_{n}(t) & =\sup \left\{\rho\left(x_{0}, x_{n}\right): \exists\left(x_{0}, \cdots, x_{n}\right) \in D^{n+1} \text { such that } \rho\left(x_{k}, x_{k+1}\right)\right. \\
& \leqq t \text { for } k=0,1, \cdots, n-1\} .
\end{aligned}
$$

Then we have:

$$
C_{n}(t)=\frac{(1+t)^{n}-(1-t)^{n}}{(1+t)^{n}+(1-t)^{n}}<1 .
$$

We will need the following lemma, (see [2]).

Lemma. Let $\varepsilon>1$ and $\delta_{0}<(\varepsilon-1 / \varepsilon+1)$. Then there exists $\delta<1$ such that the inequalities $\rho(x, y) \leqq \delta_{0}$ and $\rho(x, z) \geqq \delta$ imply $\rho(y, z) \geqq \rho(x, z)^{s}$.

C. Family $H$. Next we define family $H$, which will be central to this woxk.

Definition 1. We denote by $H$ the family of the inner functions $B$ such that for every $\theta \in] 0,1\left[\right.$ there exists $\delta_{B, \theta}<1$ such that the inequality $\rho(x, y) \leqq \delta_{B, \theta}$ holds for every pair of points $x$ and $y$ belonging to the same connected component of the set

$$
\Sigma_{B, \theta}=\{z \in D:|B(z)|<\theta\} .
$$

Sets such as $\Sigma_{B, \theta}$ or its complement have already been used; for example see [1].

The author arrived at the above definition after some discussions with S. Negrepontis.

The following preliminary results are related to the family $H$.

1. For every $B \in H$ and $\theta \in] 0,1$ [ the closure of each component of $\Sigma_{B, \theta}$ is included in $D$.

2. For $B \in H$ and $\theta \in] 0,1$, each component of the set $\Sigma_{B, \theta}$ contains at least one zero of $B$ and not more than $\log \theta / \log \delta_{B, \theta}$.

For the last statement, it is enough to observe that, if $N$ is the number of zeros in some component of $\Sigma_{B, \theta}$ and $z$ is a point of its boundary, then $\theta=|B(z)| \leqq \delta_{B, \theta}^{N}$.

3. If $B_{1} B_{2} \in H$, then $B_{1} \in H$. 
4. Let $S$ be a nonconstant singular function and let $\theta \in] 0,1[$, then the pseudo-hyperbolic diameter of each component of the set $\Sigma_{S, \theta}=\{z \in D:|S(z)|<\theta\}$ is equal to 1 . It follows that the family $H$ contains only Blaschke products.

Proof. The first part of (4) is implied by a maximum principle argument. The second part follows from the first part and (3).

5. Let $B \in H, \theta \in] 0,1[$ and $\delta \in] 0,1[$ Then there exists $\widetilde{\delta}<1$ such that the inequality $\rho(x, y) \leqq \tilde{\delta}$ holds for every pair of points $x, y$ belonging to the same component of the set

$$
E_{B, \theta, \delta}=\{z \in D: \exists w \in D \text { with } \rho(z, w)<\delta \text { and }|B(w)|<\theta\} .
$$

Proof. Let $\theta^{\prime}=C_{4}\left[\max \left(\delta, \delta_{B, \theta}\right)\right]<1$ and let $\tilde{\delta}=\delta_{B, \theta^{\prime}}<1$. For $x, y$ belonging to the same component of $E_{B, \theta, \delta}$ there exists $A_{1}, \cdots, A_{n}$, components of $\Sigma_{B, \theta}$ such that $\rho\left(x, A_{1}\right)<\delta, \rho\left(y, A_{n}\right)<\delta$ and $\rho\left(A_{k}, A_{k+1}\right)<$ $C_{2}(\delta) \forall k=0, \cdots, n-1$. We choose $\alpha_{k} \in A_{k}$ such that $B\left(\alpha_{k}\right)=0$. Therefore, we have $\rho\left(x, \alpha_{1}\right)<\theta^{\prime}, \rho\left(\alpha_{k}, \alpha_{k+1}\right)<\theta^{\prime}$ and $\rho\left(\alpha_{n}, y\right)<\theta^{\prime}$. Because of the fact that $B\left(\alpha_{1}\right)=B\left(\alpha_{2}\right)=\cdots=B\left(\alpha_{n}\right)=0$ the segments $\left[x_{1} \alpha_{1}\right]$, $\left[\alpha_{1} \alpha_{2}\right], \cdots,\left[\alpha_{n-1}, \alpha_{n}\right]$ and $\left[\alpha_{n}, y\right]$ are contained in $\Sigma_{B, \theta^{\prime}}$. Therefore, we have $\rho(x, y) \leqq \delta_{B, \theta^{\prime}}=\widetilde{\delta}<1$.

6. Let $f, g \in H$ and let $\left.\theta_{1}, \theta_{2} \in\right] 0,1[$. Then there exists $\delta<1$, such that the inequality $\rho(x, y) \leqq \delta$ holds for every pair of points $x, y$ belonging to the same component of the set $\Sigma_{f, \theta_{1}} \cup \Sigma_{g, \theta_{2}}$.

Proof. The proof is similar to the above, we can get $\delta=\delta_{f, \theta^{\prime}}$ where $\theta^{\prime}=C_{3}\left[\max \left(\delta_{f, \theta_{1}}, \delta_{g, \theta_{2}}\right)\right]$.

2. The main result. The purpose of this section is to prove that, for every element $B$ of the family $H$, (Def. $1, \S 1, \mathrm{C}$ ), the connected component of $B$ in the space of the inner functions, contains neither proper multiples of $B$, nor proper divisors of $B$.

If a set of inner functions $A$ has the property " $d(f, A)<1 \Rightarrow$ $f \in A^{\prime \prime}$, then $A$ is open and closed as one can easily verify. It is also easy to see that for every inner function $B$, there exists a smallest set containing $B$ and possessing the above property. We will denote this set by $F_{B}$. Because $F_{B}$ is open-closed, it contains the component of $B$. Therefore, it will be sufficient for our purpose, to prove that, for every $B \in H$, the set $F_{B}$ contains neither proper multiples of $B$, nor proper divisors of $B$.

The set $F_{B}$ can also be defined as follows: 
Definition 2. For every inner function $B, F_{B}$ denotes the family of the inner functions $B^{\prime}$ such that, there is a finite set of inner functions $\left\{B_{0}=B, B_{1}, \cdots, B_{n-1}, B_{n}=B^{\prime}\right\}$ joining $B$ to $B^{\prime}$, in the sense that $\left\|B_{K}-B_{K+1}\right\|<1$ for every $K=0,1, \cdots, n-1$.

In the first step, we prove that for every $B \in H$ we have the inclusion $F_{B} \subset H$ (Prop. 1). We recall that $H$ contains only Blaschke products. $(\S 1, \mathrm{c}, 4)$.

LEMMA 1. Let $B=c \prod_{i \in I} b_{\alpha_{i}}$ be an element of $H$ and let $f$ be an inner function such that $\|B-f\|<1$. Then $f$ is a Blaschke product which can be written in the form $f=c^{\prime} \prod_{i \in I} b_{\beta_{i}}$ so that $\sup _{i \in I} \rho\left(\alpha_{i}, \beta_{i}\right)<1$.

Proof. Let $\theta$ be a number such that $\|f-B\|<\theta<1$ and let us consider the set $\Sigma_{B, \theta}=\{z \in D:|B(z)|<\theta\}$. On the set $D \backslash \Sigma_{B, \theta}$ we have the following:

$$
|B(z)| \geqq \theta>\|B-f\| \geqq|B(z)-f(z)| .
$$

The hypothesis $B \in H$ implies that the boundary of each component of $\Sigma_{B, \theta}$ lies in $D \backslash \Sigma_{B, \theta}$, so that we can apply Rouche's theorem. The conclusion is that the functions $f$ and $B$ don't vanish off $\Sigma_{B, \theta}$ and they have the same (finite) number of zeros in each component of $\Sigma_{B, \theta}$. This implies that $f$ can be written in the form $f=S \prod_{i \in I} b_{\beta_{i}}$, where $S$ is a singular function and the points $\alpha_{i}$ and $\beta_{i}$ belong to the same component of $\Sigma_{B, \theta}$ for each $i \in I$. Since $B \in H$ we have:

$$
\sup _{i \in I} \rho\left(\alpha_{i}, \beta_{i}\right) \leqq \delta_{B, \theta}<1 \text {. }
$$

It remains to be shown that $S$ is a constant. This is an immediate consequence of the inclusions $\Sigma_{S,(1-\theta / 2)} \subset \Sigma_{f,(1-\theta / 2)} \subset \Sigma_{B,(1+\theta / 2)}$ combined with $\S 1, \mathrm{c}, 4$.

This simple proof, that $S$ is constant, was suggested by $D$. Marshall.

Lemma 2. Let $B=c \prod_{i \in I} b_{\alpha_{i}}$ be an element of $H$ and let $B^{\prime}=$ $c^{\prime} \Pi_{i \in I} b_{\beta_{i}}$ be a Blaschke product such that $\sup _{i \in I} \rho\left(\alpha_{i}, \beta_{i}\right)<1$. Then $B^{\prime} \in H$.

Proof. Let $0<\theta<1$; by $\S 1, \mathrm{c}, 5$ it is enough to show that the set $\Sigma_{B^{\prime}, \theta}=\left\{z \in D:\left|B^{\prime}(z)\right|<\theta\right\}$ is included in a set of the form:

$$
E_{B, \theta_{1}, \dot{\delta}}=\left\{z \in D: \exists w \in D \text { with } \rho(z, w)<\delta \text { and }|B(w)|<\theta_{1}\right\}
$$


for some $\left.\theta_{1}, \delta \in\right] 0,1[$. Therefore, it is enough to show that for some $\left.\theta_{1}, \delta \in\right] 0,1\left[\right.$ we have $\left|B^{\prime}(z)\right| \geqq \theta$ off $E_{B, \theta_{1}, \hat{\delta}}$.

Let $\delta_{0}=\sup _{i \in I} \rho\left(\alpha_{i} \beta_{i}\right)<1$ and let $\varepsilon>1$ such that $\delta_{0}<(\varepsilon-1 / \varepsilon+1)$. By $\S 1, B$, there exists $\delta<1$ such that $\rho\left(\alpha_{i}, z\right)^{\varepsilon} \leqq \rho\left(\beta_{i}, z\right)$ for every $z$ satisfying $\rho\left(\alpha_{i} z\right) \geqq \delta$.

For the above $\delta$, for $\theta_{1}=\theta^{1 / \varepsilon}$ and for $z \notin E_{B, \theta_{1}, \delta}$ we have:

$$
\left|B^{\prime}(z)\right|=\prod_{i \in I} \rho\left(\beta_{i}, z\right) \geqq \prod_{i \in I} \rho\left(\alpha_{i}, z\right)^{\varepsilon}=|B(z)|^{\varepsilon} \geqq \theta .
$$

The following proposition is an obvious consequence of Lemmas 1 and 2.

Proposition 1. Every inner function $f$, such that $d(f, H)<1$, belongs to $H$. In consequence $F_{B} \subset H$ for every $B \in H$, and $H$ is open and closed. It follows also that for every $B \in H$ the set $F_{B}$, and the component of $B$, contain only Blaschke products.

Next we give a necessary condition for two inner functions $f$ and $B$ to belong to the same component, given that $B \in H$.

Proposition 2. Let $B=c \prod_{i \in I} b_{\alpha_{i}} \in H$ and let $f \in F_{B}$; then $f$ is a Blaschke product which can be written in the form $f=c^{\prime} \prod_{i \in I} b_{\beta_{i}}$, so that $\sup \rho\left(\alpha_{i} \beta_{i}\right)<1$. In particular this is true for every inner function $f$ belonging in the same connected component as $B$.

Proof. We only consider the case $f \in F_{B}$, because $F_{B}$ contains the component of $B$.

By the definition of $F_{B}$ we have: $B=B_{1}, \cdots, B_{n-1}, B_{n}=f$ with

$$
\left\|B_{k}-B_{k+1}\right\|<1 \text { for } k=0,1, \cdots, n-1 .
$$

Since $B_{K} \in F_{B} \subset H$, Lemma 1 implies that:

$$
B_{k}=c_{k} \prod_{i \in I} b_{\alpha_{i} k}, \quad \text { with } \quad c_{0}=c, \alpha_{i}^{0}=\alpha_{i} \text { and sup } \rho\left(\alpha_{i}^{k}, \alpha_{i}^{k+1}\right)=\tau_{k}<1 .
$$

Let $\tau=\max \left(\tau_{0}, \tau_{1}, \cdots, \tau_{n-1}\right)<1$; then we have $\sup _{i \in I} \rho\left(\alpha_{i}^{0}, \alpha_{i}^{n}\right) \leqq$ $C_{n}(\tau)<1(\S 1, \mathrm{~B})$.

Finally, let $\beta_{i}=\alpha_{i}^{n} \forall i \in I$ and let $c^{\prime}=c_{n}$.

The condition of Proposition 2 is not sufficient even for elements of $H$. To show this, we follow a suggestion of D. Marshall. Consider two sufficiently lacunary sequences $\left(\alpha_{n}\right) n=1,2, \cdots$ and $\left(\beta_{n}\right) n=$ $1,2, \cdots$ such that:

$$
\frac{1+\alpha_{n}}{1-\alpha_{n}}>0, \frac{1+\alpha_{n}}{1-\alpha_{n}} \longrightarrow 0 \text { and } \frac{1+\beta_{n}}{1-\beta_{n}}=(1-i) \frac{1+\alpha_{n}}{1-\alpha_{n}}
$$


Using Lemma 1.2 of [4] one can verify that the Blaschke products $B=\prod_{n=1}^{\infty} b_{\alpha_{n}}$ and $g=\prod_{n=1}^{\infty} b_{\beta_{n}}$ don't belong to the same component; although we have $\sup _{n} \rho\left(\alpha_{n}, \beta_{n}\right)<1, f \in H$ and $g \in H$. (The gaps of the sequences $\alpha_{n}$ and $\beta_{n}$ will assure that $f \in H$ and $g \in H$.)

We conclude this section with the following proposition, which in particular implies that the component of each element of $H$ is not invariant under multiplication by $z$.

Proposition 3. For every $B \in H$, the set $F_{B}$ contains neither proper multiples of $B$, nor proper divisors of $B$. It follows that the connected component of $B$ contains neither proper multiples of $B$, nor proper divisors of $B$. In particular $B$ and $z B$ don't belong to the same component.

Proof. It is enough to prove the statement for the set $F_{B}$. Let us suppose $G B \in F_{B}$, with $G$ inner; we will prove that $G$ is constant.

By Proposition 2 we have: $B=c \prod_{i \in I} b_{\alpha_{\imath}}$ and $G B=c^{\prime} \prod_{i \in I} b_{\beta_{\imath}}$ with $\sup _{2 \in I} \rho\left(\alpha_{i}, \beta_{i}\right)<1$.

Because $B$ divides $G B$, there exists an injective function $\phi: I \rightarrow I$ such that $\alpha_{i}=\beta_{\phi(i)} \forall i \in I$. Let $\theta=\sup _{i \in I} \rho\left(\beta_{i}, \beta_{\dot{\phi}(i)}\right)=\sup _{\imath \in I} \rho\left(\beta_{\imath}, \alpha_{\imath}\right)<$ 1. Then each segment $\left[\beta_{i}, \beta_{\phi(i)}\right], i \in I$ is contained in the set $\Sigma=$ $\Sigma_{G B, \theta}=\{z \in D:|G(z) B(z)|<\theta\}$. Therefore, $\beta_{i}$ and $\beta_{\dot{\phi}(2)}$ belong to the same component of $\Sigma$.

For each connected component $\Omega$ of $\Sigma$, the set $I_{\Omega}=\left\{i \in I: \beta_{i} \in \Omega\right\}$ is finite (because $G B \in H$ ) and invariant under the injection $\phi$. Since $I=\bigcup I_{2}$ it follows that $\phi$ is bijective. In consequence we have: $\prod_{i \in I} b_{\beta_{i}}=\prod_{i \in I} b_{\beta_{\phi(i)}}=\prod_{i \in I} b_{\alpha_{\imath}}$ and $G B=c^{\prime} \prod_{i \in I} b_{\beta_{\imath}}=c^{\prime} \prod_{i \in I} b_{\alpha_{\imath}}=$ $\left(c /{ }^{\prime} c\right) B$, which implies that $G=c^{\prime} / c=$ constant.

It remains to be shown that $F_{B}$ doesn't contain any proper divisors of $B$. Let $f \in F_{B}$ with $B=f R, R$ inner. Then $f \in H$ (Proposition 1), $B \in F_{B}=F_{f}$ and $B$ is a multiple of $f$. Then, as we have already proven, $R=(B / f)=$ constant.

3. A characterization of the elements of $H$. The purpose of this section is to characterize the elements of family $H$ (Propositions 4 and 5). We first recall that $H$ contains the finite Blaschke products. Further, we can construct other elements of $H$ using the following proposition.

Proposition 4. (1) The family $H$ is closed under multiplication and (2) Let $B_{n}, n=1,2, \cdots$ be a sequence in $H$; then the infinite product $B=\prod_{n=1}^{\infty} B_{n}$ belongs to $H$, provided that:

(i) $\lim _{n \rightarrow \infty} \inf _{B_{n}(z)=0} \prod_{m \neq n}\left|B_{m}(z)\right|=1$ 
and

(ii) For every $\theta \in] 0,1\left[\right.$ we have $\sup _{n} \delta_{B_{n}, \theta}=\delta_{\theta}<1$

(where $\delta_{B_{n}, \theta}$ denotes the supremum of the pseudo-hyperbolic diameters of the connected components of the set $\Sigma_{B_{n}, \theta}=\left\{z \in D:\left|B_{n}(z)\right|<\theta\right\}$ according to Definition 1).

Proof. (1) Let $B_{1} \in H$ and $B_{2} \in H$; we will prove that $B_{1} B_{2} \in H$. Let $\theta \in] 0,1[$; we consider the following sets:

$$
\Sigma_{1}=\Sigma_{B_{1}, \sqrt{\theta}}=\left\{z \in D:\left|B_{1}(z)\right|<\sqrt{\theta}\right\}, \Sigma_{2}=\Sigma_{B_{2}, \sqrt{\theta}}=\left\{z \in D:\left|B_{2}(z)\right| \sqrt{\theta}\right\}
$$

and $\Sigma=\Sigma_{B_{1} B_{2}, \theta}=\left\{z \in D:\left|B_{1}(z) B_{2}(z)\right|<\theta\right\}$.

We trivially have $\Sigma \subset \Sigma_{1} \cup \Sigma_{2}$ and the proof of (1) is finished by $\S 1, \mathrm{c}, 6$.

(2) Let $B_{n}=c_{n} \prod_{i \in I_{n}} b_{\alpha_{i} n}, n=1,2, \cdots$ be a sequence in $H$ satisfying (i) and (ii). Condition (i) implies the convergence of the product $B=\prod_{n=1}^{\infty} B_{n}$. We have to prove that $B \in H$.

Let $\theta \in] 0,1[$; then we consider the following sets:

$\Sigma=\{z \in D:|B(z)|<\theta\}$ and $\Sigma_{n}=\left\{z \in D:\left|B_{n}(z)\right|<\theta^{1 / 8}\right\}, \quad n=1,2, \cdots$.

By (ii) we have $\sup _{n} \delta_{n, \theta}=\delta_{\theta}=\delta<1$.

Let $\varepsilon>1$ such that $\delta<(\varepsilon-1 / \varepsilon+1)$; by $\S 1$, B there exists $\left.\theta^{\prime} \in\right] \theta^{1 / 8}$, 1 [ such that the relations $\rho\left(z, z^{\prime}\right) \leqq \delta$ and $\rho(\alpha, z) \geqq \theta^{\prime}$ imply $\rho\left(\alpha, z^{\prime}\right) \geqq$ $\rho(\alpha, z)^{\varepsilon}$.

This fact combined with condition (i) implies that there exists $n_{0}$ such that $\left|\prod_{m \neq n} B_{m}(z)\right| \geqq \theta^{\prime} \geqq \theta^{1 / 8}$ for every $n>n_{0}$ and $z \in \Sigma_{n}$. This last inequality also holds on the boundary of each component of $\Sigma_{n}\left(n>n_{0}\right)$, where we have $\left|B_{n}(z)\right|=\theta^{1 / 8}$. Therefore, on the boundary of each component of $\Sigma_{n}\left(n>n_{0}\right)$ we have

$$
|B(z)|=\left|B_{n}(z)\right|\left|\prod_{m \neq n} B_{m}(z)\right| \geqq \theta^{1 / 8} \theta^{1 / 8}=\theta^{1 / 4} .
$$

It follows that each component of $\Sigma$, containing at least one zero of $\Pi_{n>n_{0}} B_{n}$, is included in some connected component of some $\Sigma_{n}\left(n>n_{0}\right)$. Therefore, its pseudo-hyperbolic diameter is less than or equal to $\delta<1$.

It remains to control the pseudo-hyperbolic diameters of the other components of $\Sigma$, that is of the components of $\Sigma$ containing some zeros of $B_{1} B_{2} \cdots B_{n_{0}}$.

Let us consider the set $K=\left\{z \in D:\left|B(z) \cdots B_{n_{0}}(z)\right|<\theta^{1 / 8}\right\}$. By the first part of Proposition $4, B_{1} B_{2} \cdots B_{n_{0}} \in H$ and therefore, the pseudo-hyperbolic diameters of the components of $K$ are less than $\delta^{\prime}<1$. 
We first observe that $K \cap\left(\mathbf{U}_{n>n_{0}} \Sigma_{n}\right)=\phi$; indeed, on $\Sigma_{n}\left(n>n_{0}\right)$ we have the relation $\left|B_{1}(z) \cdots B_{n_{0}}(z)\right| \geqq\left|\prod_{m \neq n} B_{n}(z)\right| \geqq \theta^{1 / 8}$ and on $K$ we have the opposite strict inequality.

The fact that, $K$ and $\bigcup_{n>n_{0}} \Sigma_{n}$ are disjoint, combined with a maximum principle's argument implies $\left|\Pi_{n>n_{0}} B_{n}(z)\right| \geqq \theta^{1 / 4}$ on $K$ and on the boundary of each component of $K$, where we also have $\left|B_{1}(z) \cdots B_{n_{0}}(z)\right|=\theta^{1 / 8}$. We deduce that on the boundary of each component of $K$ we have $|B(z)| \geqq \theta^{1 / 8} \theta^{1 / 4}=\theta^{3 / 8}>\theta$. It follows that each component of $\Sigma$ containing some zeros of $B_{1} B_{2} \cdots B_{n_{0}}$ is included in $K$. Therefore, its pseudo-hyperbolic diameter is less than $\delta^{\prime}$. We conclude that the pseudo-hyperbolic diameters of the components of $\Sigma$ are less than or equal to $\tilde{\delta}=\max \left(\delta, \delta^{\prime}\right)<1$, and the proof is finished.

Next we prove that every element of $H$ can be represented as a product of finite Blaschke products, in the sense of Proposition 4. Thus, we have an obvious characterization of the elements of $H$.

Proposition 5. Let $B=\prod_{k=1}^{\infty} b_{\alpha_{k}}$ be an infinite Blaschke product belonging to the family $H$. Then, there exists a sequence $B_{n}, n=$ $1,2, \cdots$ of finite Blaschke products, such that:

(i) $\lim _{n} \inf _{B_{n}(z)=0} \prod_{m \neq n}\left|B_{m}(z)\right|=1$.

(ii) $\sup _{n} \delta_{B_{n}, \theta}<1$ for every $\left.\theta \in\right] 0,1[$ and

(iii) $B=\prod_{n=1}^{\infty} B_{n}$.

Proof. Let $\theta_{n}=1-(1 / n+1), n=1,2, \cdots$, let

$$
\Sigma_{B, \theta_{n}}=\left\{z \in D:|B(z)|<\theta_{n}\right\}
$$

and let $A_{n}=\left\{k: \alpha_{k}\right.$ belongs to the same component of $\Sigma_{B, \theta_{n}}$ as $\left.\alpha_{1}\right\}$.

Finally, we consider the following Blaschke products:

$$
B_{1}=\prod_{k \in A_{1}} b_{\alpha_{k}} \text { and } B_{n}=\prod_{k \in A_{n} \backslash A_{n-1}} b_{\alpha_{k}} \text { for } n \geqq 2 .
$$

The functions $B_{n}, n=1,2, \cdots$ are finite Blaschke products because the sets $A_{n}$ are finite $(\S 1, \mathrm{c}, 2)$. We also have $B=\prod_{n=1}^{\infty} B_{n}$, because $\theta_{n}$ is increasing and converges to 1 .

Let $\theta \in] 0,1\left[\right.$; then $\left\{z \in D:\left|B_{n}(z)\right|<\theta\right\}=\Sigma_{B_{n}, \theta} \subset \Sigma_{B, \theta}$, because $B_{n}$ divides $B$. Therefore, $\delta_{B_{n}, \theta} \leqq \delta_{B, \theta}<1$ and condition (ii) is verified.

Let $z$ be a zero of $B_{n+1}$. By the maximum modulus principle we have $\left|\prod_{m \neq n+1} B_{m}(z)\right| \geqq \min \left(\theta_{n}, \theta_{n+1}\right)=\theta_{n} \rightarrow 1$ and condition (i) is also verified. Therefore, the proof is complete now.

At this point, I wish to express my thanks to D. Marshall for his help with Proposition 5.

Because the simple Blaschke factors $c b_{\alpha}(|c|=1,|\alpha|<1)$ belong 
to $H$, using Proposition 4 we observe that the following families are contained in $H$ :

$$
\Lambda^{1}=\left\{c \prod_{n=1}^{\infty} b_{\alpha_{n}} ; \lim _{n} \prod_{m \neq n}\left|\frac{\alpha_{n}-\alpha_{m}}{1-\bar{\alpha}_{n} \alpha_{m}}\right|=1\right\}
$$

and $\Lambda^{n}=\left\{B_{1} B_{2} \cdots B_{n} ; B_{1}, B_{2}, \cdots, B_{n} \in \Lambda^{1}\right\}, n \geqq 2$.

These families are also open-closed. One way to see it is the following.

We say that a subfamily $\Lambda$ of $H$ is saturated if $c \prod_{i \in I} b_{\alpha_{i}} \in \Lambda$ and $\sup _{i \in I} \rho\left(\alpha_{i}, \beta_{i}\right)<1$ imply $c^{\prime} \prod_{i \in I} b_{\beta_{i}} \in \Lambda$.

Lemma 1 implies that every saturated subfamily of $H$ is openclosed. Therefore it is enough to show that $\Lambda^{n}(n \geqq 1)$ is saturated. Using the above technique one can prove that the family of the product (in the sense of Proposition 4) of the elements of saturated subfamilies of $H$ is also a saturated subfamily of $H$. Starting from the family of the simple Blaschke factors $\Lambda=\left\{c b_{\alpha} ;|c|=1,|\alpha|<1\right\}$, which is obviously saturated, one concludes that the families $\Lambda^{n}, n \geqq 1$ are saturated, and therefore open-closed.

Finally we mention that $H \neq \bigcup_{n=1}^{\infty} \Lambda^{n}$. For $B \in H$ and $\left.\left.\theta \in\right] 0,1\right]$ we denote $N_{B, \theta}$ the limes inferior of the number of zeros of $B$ in each component of $\Sigma_{B, \theta}$ (where the limes inferior is taken over all components of $\Sigma_{B, \theta}$ ). For $B \in \Lambda^{n}$ one can see that $N_{B, \theta} \leqq n$. Therefore, for $B \in \bigcup_{n=1}^{\infty} \Lambda^{n}$ we have: $\sup _{0<\theta<1} N_{B, \theta}<\infty$.

In consequence, it is enough to give an example of $B \in H$ for which $\sup _{\theta} N_{B, \theta}=\infty$. For $n \in Z=\{0, \pm 1, \pm 2, \cdots\}$ let $\beta_{n}$ such that $\left(1+\beta_{n}\right) /\left(1-\beta_{n}\right)=1+i n$. For every $\left.\theta \in\right] 0,1[$ there exists a natural number $M=M_{\theta}$ such that for every $n \in Z$ we have $\Pi_{|n-m| \geqq M}\left(\beta_{n}, \beta_{m}\right) \geqq$ $\theta$. Consider $\theta_{n}=1-1 /(n+1)$ and let $M_{n}=M_{\theta_{n}}$.

Consider also the following inductively defined sequence $S_{n}$ of subsets of $Z$ :

$$
S_{1}=\{0\} . \text { Suppose } S_{1}, S_{2}, \cdots, S_{k} \text { are defined . }
$$

Denote by $\sigma_{k}$ the maximum element of $S_{k}$ and define $S_{k+1}$ as follows:

$$
S_{k+1}=2 M_{k}+\sigma_{k}+\left[\bigcup_{\lambda=1}^{k} S_{\lambda}\right]=\left\{x+2 M_{k}+\sigma_{k} ; x \in \bigcup_{\lambda=1}^{k} S_{k}\right\} .
$$

Each $S_{k}$ is finite. Set $B_{k}=\prod_{n \in S_{k}} b_{\beta_{n}}$ and $B=\prod_{k=1}^{\infty} B_{k}$. It is true that $B \in H$, (use for example Proposition 4); but $N_{B, \theta_{n}} \vec{n}^{\infty}$. Therefore $B \notin \bigcup_{n=1}^{\infty} \Lambda^{n}$ and $H \neq \bigcup_{n=1}^{\infty} \Lambda^{n}$.

\section{REFERENCES}

1. S.-Y. Chang, A characterization of Douglas subalgebras, Acta Math., 137 (1976), 81-86. 
2. J. P. Earl, On the interpolation of bounded sequences by bounded functions, J. London Math. Soc., (2), 2 (1970), 544-548.

3. K. Hoffman, Banach spaces of analytic functions.

4. D. Herrero, Inner functions under uniform topology, Pacific J. Math., 51 (1974), 167-175.

5. — Inner functions under uniform topology II, Revista de la Union Matematica Argentina, 28 (1976).

6. V. Nestoridis, Fonctions Interieures: Composantes Connexes et Multiplication par Un Produit de Blaschke, These de Trosieme cycle, Grenoble.

7. — Inner functions: Invariant connected components, Pacific J. Math., 83 (1979), 473-480.

8. W. Rudin, Real and complex analysis.

Received November 21, 1978 and in revised form June 21, 1979.

UNIVERSITY OF WASHINGTON

Seattle, WA 98195 



\section{PACIFIC JOURNAL OF MATHEMATICS}

\section{EDITORS}

DONALD BABBITT (Managing Editor)

University of Galifornia

Los Angeles, California 90024

HUGo RossI

University of Utah

Salt Lake City, UT 84112

C. C. MOORE AND ANDREW OGG

University of California

Berkeley, CA 94720

\section{J. DUGUNDJI}

Department of Mathematics University of Southern California Los Angeles, California 90007

R. FINN AND J. MILGRAM Stanford University Stanford, California 94305

ASSOCIATE EDITORS
E. F. BECKENBACH
B. H. NeumanN
F. WoLF
K. YosHIDA

\section{SUPPORTING INSTITUTIONS}

UNIVERSITY OF BRITISH COLUMBIA UNIVERSITY OF SOUTHERN CALIFONIA CALIFORNIA INSTITUTE OF TECHNOLOGY UNIVERSITY OF CALIFORNIA MONTANA STATE UNIVERSITY STANFORD UNIVERSITY UNIVERSITY OF HAWAII UNIVERSITY OF TOKYO UNIVERSITY OF NEVADA, RENO UNIVERSITY OF UTAH NEW MEXICO STATE UNIVERSITY WASHINGTON STATE UNIVERSITY OREGON STATE UNIVERSITY UNIVERSITY OF OREGON UNIVERSITY OF WASHINGTON 


\section{Pacific Journal of Mathematics \\ Vol. 87, No. $1 \quad$ January, 1980}

Spiros Argyros, A decomposition of complete Boolean algebras ..........

Gerald A. Beer, The approximation of upper semicontinuous multifunctions

by step multifunctions . . . . ....................

Ehrhard Behrends and Richard Evans, Multiplicity theory for Boolean

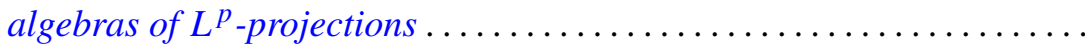

Man-Duen Choi, The full $C^{*}$-algebra of the free group on two

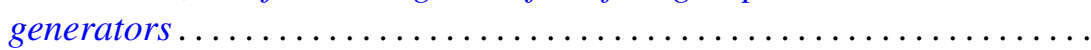

Jen-Chung Chuan, Axioms for closed left ideals in a $C^{*}$-algebra . . . . . . . .

Jo-Ann Deborah Cohen, The strong approximation theorem and locally

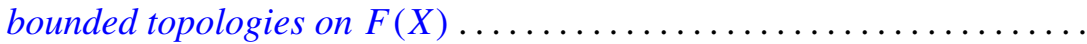

Eugene Harrison Gover and Mark Bernard Ramras, Increasing sequences of Betti numbers............................

Morton Edward Harris, Finite groups having an involution centralizer with

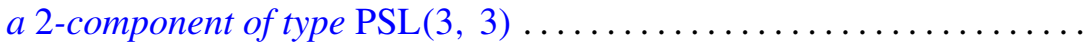

Valéria Botelho de Magalhães Iório, Hopf $C^{*}$-algebras and locally compact

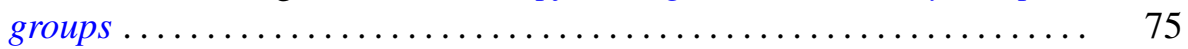

Roy Andrew Johnson, Nearly Borel sets and product measures . . . . . . . . . .

Lowell Edwin Jones, Construction of $Z_{p}$-actions on manifolds . . . . . . . . .

Manuel Lerman and Robert Irving Soare, $d$-simple sets, small sets, and

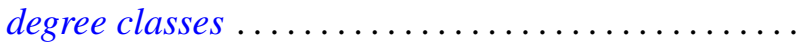

Philip W. McCartney, Neighborly bushes and the Radon-Nikodým property

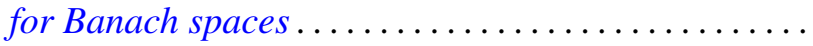

Robert Colman McOwen, Fredholm theory of partial differential equations on complete Riemannian manifolds.

Ernest A. Michael and Carl Preston Pixley, A unified theorem on continuous selections.

Ernest A. Michael, Continuous selections and finite-dimensional sets .

Vassili Nestoridis, Inner functions: noninvariant connected components...

Bun Wong, A maximum principle on Clifford torus and nonexistence of proper holomorphic map from the ball to polydisc.

Steve Wright, Similarity orbits of approximately finite $C^{*}$-algebras . . .

Kenjiro Yanagi, On some fixed point theorems for multivalued

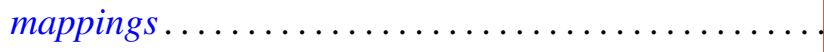

Wieslaw Zelazko, A characterization of LC-nonremovable ideals in commutative Banach algebras 